\title{
Epidemiology of Knee Injuries in Indian Kabaddi Players
}

\author{
Mandeep Singh Dhillon, ${ }^{1}$ Rakesh John, ${ }^{1,}$ Siddhartha Sharma, ${ }^{1}$ Sharad Prabhakar, ${ }^{1}$ Prateek Behera, ${ }^{1}$ \\ Soumya Saxena, ${ }^{2}$ Heera Singh, ${ }^{3}$ and Devendra Chouhan ${ }^{4}$ \\ ${ }^{1}$ Department of Orthopaedics, PGIMER, Chandigarh, India \\ ${ }^{2}$ Department of Physical Medicine and Rehabilitation, PGIMER, Chandigarh, India \\ ${ }^{3}$ Department of Immunopathology, PGIMER, Chandigarh, India \\ ${ }^{4}$ PGIMER, Chandigarh, India \\ "Corresponding author: Rakesh John, Department of Orthopaedics, PGIMER, Chandigarh, India. Tel: +919781335111, E-mail: rakeshjohn23@gmail.com
}

Received 2015 October 31; Revised 2015 December 14; Accepted 2015 December 15.

\begin{abstract}
Background: Detailed injury epidemiology is an unmatched tool for reducing injury risks associated with any sport. Very little epidemiologic data exists regarding injuries sustained by kabaddi players.

objectives: To study knee injuries in kabaddi players.

Patients and Methods: This is a cross-sectional, observational study. Seventy six kabaddi players (both professionals and amateurs) presenting with sports-related knee injuries were identified from previous records; data was analysed for demographic features, injury mechanism, injury scenario, duration of injury before presentation, injury pattern, management, return to sport and time lost in sport due to the knee injury.

Results: The mean duration prior to presentation was 14.4 months. Majority of the injuries (88.16\%) occurred during competitive games. The most common injury noted was ACL tear (89.47\%), followed by meniscus tears which were noted in $68.42 \%$ of the players. Forty four players (57.89\%) retired from the sport as a consequence of the knee injury. Time lost in sport in those who returned to kabaddi was an average of 16.6 months; signifying the high morbidity due to knee injuries in kabaddi players. Return to sport was significantly more $(\mathrm{P}=0.04)$ in those treated surgically than in those treated conservatively; but was not affected by mechanism of injury, injury scenario, injury pattern, duration of injury at presentation or level of competition of the athlete.

Conclusions: Epidemiologic studies in kabaddi are the need of the hour to assess the burden of this predominantly rural sport and to implement injury prevention programs. Our study emphasises the morbidity of knee injuries in kabaddi players of India and the exacting toll it takes on their careers.
\end{abstract}

Keywords: Kabaddi, knee injuries, ACL Tear, Meniscus Tear, Sports-Related Injuries

\section{Background}

Kabaddi is a popular contact sport/wrestling sport which has its origins in South Asia and has spread to Iran, Japan, Southeast Asia, United Kingdom, Canada etc. A highly popular sport in India, it's the state game of Tamil Nadu, Karnataka, Punjab, Maharashtra, Bihar, Telangana and Andhra Pradesh. It is also the national game of Bangladesh and Nepal (1). Internationally, the game has been a part of the Asian games since 1990 and has an annual World cup event for both men and women apart from various professional leagues and other international tournaments (1).

Although the game has gained tremendous popularity in the world, there is a dearth of epidemiological studies in the sport. Detailed injury epidemiology is an invaluable tool for reducing injury risks associated with any sport. Although knee injuries are one of the commonest injuries sustained by kabaddi players, no study has been done to date focussing on the epidemiology of knee in- juries in these players (2-4).

\section{Objectives}

Present study was aimed to investigate knee injuries in kabaddi players.

\section{Patients and Methods}

This was a cross-sectional study conducted at the Sports injury clinic of post graduate institute of medical education and research, Chandigarh. Duration of study was from April 2010 to December 2014. The study was approved by the institute ethics committee (intramural), PGIMER, Chandigarh. An informed consent was taken from all the participants. Sports-related knee injuries were identified from previous records. 83 kabaddi players presenting with knee injuries to the clinic during this duration were enrolled into the study. Seven players were excluded (reasons being incomplete data in 4 players and 
non-kabaddi injury in 3). Overall 76 kabaddi players were included for the final analysis.

\subsection{Selection Criteria}

Inclusion criteria were: kabaddi players aged between 18 to 45 years diagnosed with menisco-ligamentous knee injury, caused or symptomatic during sports practice, which prevented the athlete from playing the sport completely or for a variable period of time.

Exclusion criteria were: athletes who did not have a confirmed diagnosis (either clinical or by imaging) and those who had knee injury not related to sports (for example, kabaddi players with ACL tears caused by road traffic accidents). Athletes with patellofemoral pain syndrome, chondral injuries, extensor mechanism injuries, minor injuries/sprains etc. were excluded.

\subsection{Data Extraction and Synthesis}

A standardised protocol was used to collect information about the demographics and knee injuries sustained by the players at the time of presentation to the clinic. Return to sport and time lost in sport due to the injury was separately enquired over a telephonic interview.

The athletes were classified into professional and amateur groups depending on the level of their playing field. Any player playing for a professional club or representing state/country was considered as a professional athlete. Other athletes including inter-collegiate players were grouped into amateur category for the purpose of this study.

The mechanism of injury was classified into contact and non-contact mechanisms according to the classification system of Marshall et al. (5) which is based on external force application. Contact injuries were further classified into direct and indirect contact mechanisms. Direct contact to the knee is classified as a direct contact mechanism of injury, whereas direct contact to the athlete, but not directly to the injured knee is classified as an indirect contact mechanism of injury. A non-contact injury is the result of the athlete's own movements and does not involve contact with another athlete or object (5).

The injury scenario specifically related to match time or practice time injury was documented. The duration between injury and first presentation to our clinic was noted. Return to sport was assessed and the time lost in sport (in months) as a consequence of the injury was noted in those athletes who returned to sport.

The knee injuries were categorized as ACL, MCL, LCL, PCL, medial meniscus and lateral meniscus tears. All the diagnoses were confirmed by imaging (both plain radiographs and MRI which were reported by radiologist) and $68.4 \%$ of the diagnoses were confirmed by arthroscopy.
The type of management, whether surgical or conservative, was noted. Not all athletes who were treated surgically were operated at our center. Some athletes $(n=4)$ chose to undergo surgical treatment at a different institute after initial consultation at our institute but even these athletes were followed up over telephone regarding their activity levels, rehabilitation protocols and return to active sports post-surgery.

\subsection{Statistical Analysis}

Categorical data was analysed using Chi-square test and Fischer's exact test whereas independent sample t-test was used for continuous data. Correlations between variables were also calculated and $\mathrm{P}<0.05$ was considered to be statistically significant. SPSS version 21 (software package used for statistical analysis) was used for the data analysis.

\section{Results}

\subsection{Demographic Data}

The mean recorded age at presentation was 25.9 years (Range: 23 - 36 years; $S D=6.8$ ). Seventy five out of the 76 athletes were males and only 1 athlete was a female player. In 40 patients, the left knee was affected and in the rest 36 patients the right knee was affected. Players from rural areas constituted $85.5 \%$ of the population $(n=65)$ whereas only 11 out of the 76 players hailed from urban areas.

The mean height and weight of the athletes was $1.81 \mathrm{~m}$ $(\mathrm{SD}=6.64)$ and $79.91 \mathrm{~kg}(\mathrm{SD}: 8.94)$ respectively. The mean BMI was 24.45 (median $=24.35 ; \mathrm{SD}=2.24$ ). Thirty seven athletes had a normal BMI whereas 21 were overweight (BMI = 25 - 30). No significant difference was noted in the BMI of professional and amateur athletes (24.43 and 24.49 respectively).

\subsection{Professional vs. Amateur}

Forty-one athletes (53.9\%) were professional players and this group included a few players who represented the Indian national team at international events. Thirty-five were amateur players, which included inter-collegiate athletes and also those who played the sport just as a hobby.

\subsection{Mechanism of Injury}

Fifty-five players injured their knees via a contact mechanism (72.37\%). Non-contact mechanism of injury was noted in the other $27.63 \%$ of the athletes $(n=21)$. Contact injuries were significantly higher in kabaddi players than non-contact injuries $(\mathrm{P}<0.0001)$.

On further analysis, $72.73 \%$ of the contact injuries were by direct contact mechanism $(n=40)$ with indirect contact mechanism accounting for the other $27.27 \%(n=15)$. 


\subsection{Injury Scenario}

Sixty-seven athletes sustained their knee injuries during match time (88.16\%) whereas 9 athletes sustained injury during practice time (11.84\%) which was highly significant $(\mathrm{P}<0.0001)$

\subsection{Duration of Injury at Presentation}

The duration between injury and presentation at our center had a mean of 14.4 months (Range 1 - 60 months). Only 14 athletes presented within the first 3 months after the injury while 18 athletes presented between 4 to 6 months, 6 athletes presented between 7 to 9 months, 20 athletes presented between 10 to 12 months and 18 players presented more than 12 months after the index injury (see Figure 1)

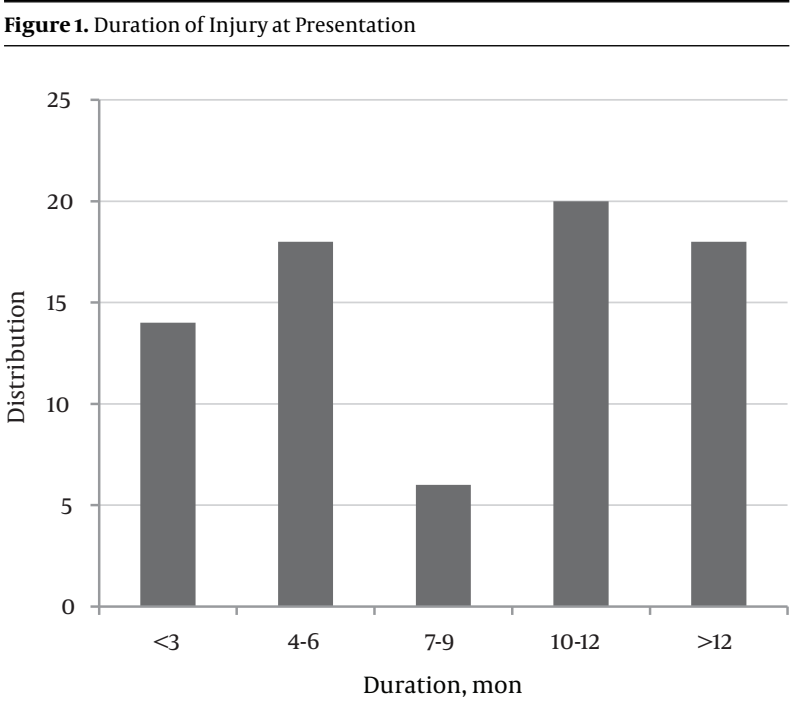

Bar graph showing the variable distribution of injury duration at presentation among the players.

\subsection{Injury Pattern}

The most common injury noted among the players was ACL tear. ACL injury was sustained by $89.47 \%$ of the players i.e. 68 out of 76 players among which 66 were complete and 2 were partial tears.

Meniscus injuries were sustained by $68.42 \%$ of the players; 32 were medial meniscus tears and 20 were lateral meniscus tears. Twenty-one players sustained a medial collateral ligament (MCL) injury (27.63\%) and 7 players had a lateral collateral ligament (LCL) injury. Only 5 players sustained a PCL injury which was the least common injury noted among these players (see Figure 2). These injuries occurred in varying combinations and the most common combination of injuries were ACL tears combined with medial meniscus tears which was seen in $40 \%$ of the athletes.

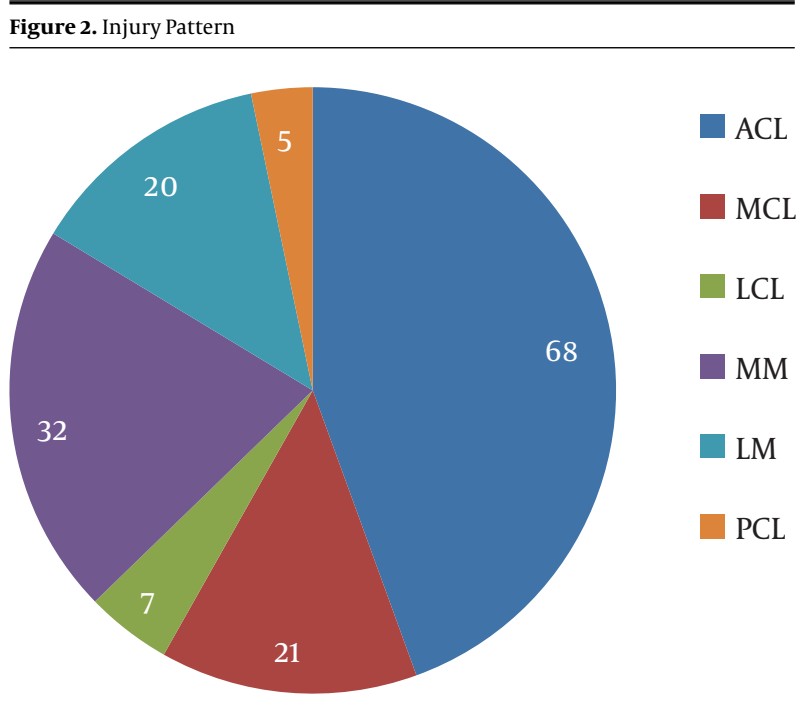

Pie chart showing the distribution of various injuries among the players.

\subsection{Management}

Fifty two of the players opted for surgical management (68.42\%) whereas 24 players were treated conservatively by physiotherapy with/without bracing. Nineteen of these 24 players chose conservative management over surgical management due to their personal reasons whereas the other 5 were advised conservative management as their injuries were not severe enough to merit surgery.

\subsection{Return to Sport and Time Lost in Sport}

Forty-four players stopped playing kabaddi altogether as a consequence of their knee injuries (57.89\%). Only 32 players returned to playing Kabaddi (42.11\%). In those athletes who returned to playing the sport, the time lost from active sports was an average of 16.6 months (Range $=7$ to 36 months).

Return to sport was higher in the professional players compared to the amateur players. In the former, 51.21\% players returned to sport ( $n=21 / 41$ players) whereas in the latter group, $31.43 \%$ of the players returned to sport $(\mathrm{n}=$ 11/35 players). Although professional athletes showed an increased tendency to return to sport, these results were not statistically significant $(\mathrm{P}=0.082)$.

When return to sport was analyzed according to the mode of management, return to sport was significantly higher in those who were surgically treated than those who were treated conservatively $(\mathrm{P}=0.04)$. It was noted that $81.8 \%$ of the athletes who returned to sport were treated surgically $(n=26 / 32)$ and the rest were treated conservatively. 


\section{Discussion}

There is limited research on sports injury epidemiology from South Asia, particularly in India (2-4). Very few studies exist on the epidemiology of injuries in kabaddi players or for that matter in any sport in India (2-4). The level of evidence of the current literature is also low as most of these studies are results of surveys which were poorly designed (Table 1$)(3,4)$.

Kabaddi is an ancient and popular sport in Asia whose scope and popularity is increasing worldwide day by day. However, no research focussed on the epidemiology of knee injuries in kabaddi players has been done to date, although knee injuries are one of the commonest injuries sustained by kabaddi players $(2,4)$.

Kabaddi remains a predominantly male-dominated sport with $98.68 \%$ of the injured players in this study being male. It is chiefly a rural sport with $85.5 \%$ of the players being from rural areas. The rural background of the sport and the associated lack of knowledge, awareness and health consciousness in rural areas maybe the reason for the high mean duration between injury and presentation (mean $=14.4$ months after initial injury). Kabaddi players are more likely to sustain an injury during a competitive game rather than during practice sessions $(\mathrm{P}<0.0001)$ which might be due to the increased level of competitiveness during the match.

Majority of the players (57.89\%) are forced to leave the sport due to the knee injury with amateurs giving up the sport more readily. Only $42.11 \%$ of the players returned to sport. In players who presented within 6 months of injury, the return to sport percentage goes up to $50 \%$. In our observations, return to sport was significantly higher in those who were operated than those treated conservatively $(\mathrm{P}=$ 0.04 ) but was not associated with the injury duration, injury scenario, competitive level and type of injury.

The most common knee injury noted in these players was ACL injury (89.47\%). This observation stands out differently from other contact sports like American football, Australian rules football, Rugby etc. where the MCL is the most common ligament to be injured (6-8). There are probably 3 reasons for this observation. The first reason may be due to the fact that initial documentation of these predominantly rural setting injuries was poor. Secondly, MCL injuries in isolation have a tendency to heal without much residual disability, and many such cases may not present at a tertiary care center like ours. Another reason might be that in kabaddi, chances of direct twisting injuries to the knee are higher unlike other similar contact sports. Defenders frequently tackle the raider by holding his lower extremity to prevent him from reaching back to his corner wherein the chances of inadvertently twisting the knee with an undue force is very high.

Prabhu et al. (3) noted in a survey that knee dislocation is the most common knee joint injury in kabaddi (70\%) followed by ligament injuries (30\%). These ligament injuries and knee dislocations were not further subclassified. Their findings are in stark disparity with our results where $\mathrm{ACL}$ injuries were the most common injury (89.47\%) and no case of knee dislocation was observed. This may also be a reflection of the fact that most of our injuries were chronic presentations or referrals. It is pertinent to note that return to sport in kabaddi players has never been assessed in any previous study.

It is commonly recognized that ACL tear represents a significant risk to a sportsperson's career across many sports (9-11). In order to reduce the incidence of ACL tears in kabaddi players, a high volume of weight training to increase strength, as well as proprioceptive exercises integrated into the training programs, are needed (12). In addition, some education of trainers, coaches and athletes should be incorporated into professional and semiprofessional leagues.

PCL injuries were noticeably rare, with only 5 cases being documented. PCL tears are commonly misdiagnosed, often being classified as a minor sprain or may remain undiagnosed $(8,13)$. This may indeed be the case in this study, despite specific injury diagnostic categorization. After all, the nature of kabaddi produces frequent episodes of landing heavily on the flexed knee and forced hyperextension. However, some players may either cope well with a minor degree of chronic PCL injury and the injury usually heals satisfactorily unlike the $\mathrm{ACL}(8,13)$.

Our study is not only the first epidemiologic study of knee injuries in kabaddi players but also the study with the largest study population of kabaddi players to date (2). This study brings to light the enormity of the burden of knee injuries in kabaddi players and the harsh toll it takes on the players' careers.

We acknowledge the limitations of this study. Being a non-randomised, observational study, it is associated with an inherent bias in patient selection. However, when observational studies provide the only data or best data available, they can significantly influence clinical practice (14). Four patients chose to undergo surgery at a different institute due to long waiting lists which might have affected the outcomes and finally return to sport. However, these patients were a small percentage and the protocol of management at these institutes was similar to our institute and hence the risk of bias is low. Another significant limitation is that this study is a hospital-based study and hence we were unable to neither calculate incidence rates of different injuries nor identify risk factors for knee injury. Prospective cohort studies in different kabaddi 
Table 1. Summary of Existing Literature on the Epidemiology of Injuries in Kabaddi Players Compared With Data From our Study

\begin{tabular}{|c|c|c|c|c|c|c|}
\hline Authors & Study Design & Country & No. of Players & Most Common Injury & Follow-Up & Return to Sport \\
\hline Moeini et al. (2) & Cross-sectional study & Iran & 73 & Muscular injuries & No & - \\
\hline Prabhu et al. (3) & Survey & India & 30 & Ankle $>$ knee & No & - \\
\hline Kurup et al. (4) & Survey & India & Not defined & Knee & No & - \\
\hline Our study & Cross-sectional study & India & 76 & Knee (ACL) & Yes & Calculated \\
\hline
\end{tabular}

leagues/clubs and tournaments are needed to better define the risk factors for injury and incidence rates of different injuries in kabaddi players.

The current study supports existing previous research that the knee joint is one of the most commonly injured joints in kabaddi (4). It highlights the fact that the morbidity associated with knee injuries in kabaddi is high and strategies need to be considered and implemented to reduce this morbidity.

A high proportion of ACL injuries were noted in kabaddi players which probably accounts for the heavy toll on the players' careers as ACL tears are known to have a significant detrimental effect on a sportsperson's career. Although this summary of the considerable impact of ACL tear is not new, it serves as a reminder of the need to continue to perfect our understanding, prevention, and management of the injury. Enhanced preventive protocols are warranted more than ever in the game of kabaddi.

Long-term, prospective studies with associated exposure data and video footage are needed in the future to precisely define injury incidence and mechanisms. This will assist in avoiding inappropriate decision-making on issues such as game rules and training protocols based on the offthe-cuff observation of groups of injuries with inadequate understanding of their ensuing impact.

\section{Footnote}

Authors' Contribution: Study concept and design: Mandeep Singh Dhillon; acquisition of data: Rakesh John, Siddhartha Sharma, Prateek Behera, Heera Singh, Soumya Saxena; analysis and interpretation of data: Rakesh John, Mandeep Singh Dhillon; drafting of the manuscript, Rakesh John, Mandeep Singh Dhillon; critical revision of the manuscript for important intellectual content, Mandeep Singh Dhillon; statistical analysis, Rakesh John, Siddhartha Sharma; administrative, technical, and material support; Sharad Prabhakar, Devendra Chouhan; study supervision, Mandeep Singh Dhillon.

\section{References}

1. Sudhakar HH, Majumdar P, Umesh V, Panda K. Second to fourth digit ratio is a predictor of sporting ability in elite Indian male kabaddi players. Asian JSports Med. 2014;5(3):ee23073. doi:10.5812/asjsm.23073. [PubMed: 25520769].

2. Moeini SM, Hojat S, Aghaei R. The epidemiology of some common injuries in elite male kabaddi player. Sport sciences quarterly. 2011;2(6):1130.

3. Prabhu A, Kishore K. Common injuries among kabaddi and kho-kho players-an empirical study. Int J Engg Res Sports Sci. 2014;1(7):1-4.

4. Kurup VKM, Chowdhary A. Injury Spectrum of Amateur CollegeGoing Athletes in Southern India -A Survey. Int Res J Medical Sci. 2014;2(9):20-1.

5. Marshall SW, Padua D, McGrath M. In: Understanding and preventing noncontact ACL injuries. Hewett TE, Schultz SJ, Griffin LY, editors. ; 2007.

6. Meyers MC, Barnhill BS. Incidence, causes, and severity of high school football injuries on FieldTurf versus natural grass: a 5-year prospective study. Am J Sports Med. 2004;32(7):1626-38. [PubMed: 15494326].

7. Orchard J, Seward H. Epidemiology of injuries in the Australian Football League, seasons 1997-2000. Br J Sports Med. 2002;36(1):39-44. [PubMed: 11867491].

8. Dallalana RJ, Brooks JH, Kemp SP, Williams AM. The epidemiology of knee injuries in English professional rugby union. Am J Sports Med. 2007;35(5):818-30. doi: 10.1177/0363546506296738. [PubMed: 17293461].

9. Joseph AM, Collins CL, Henke NM, Yard EE, Fields SK, Comstock RD. A multisport epidemiologic comparison of anterior cruciate ligament injuries in high school athletics. J Athl Train. 2013;48(6):810-7. doi: 10.4085/1062-6050-48.6.03. [PubMed: 24143905].

10. Arendt E, Dick R. Knee injury patterns among men and women in collegiate basketball and soccer. NCAA data and review of literature. Am J Sports Med. 1995;23(6):694-701. [PubMed: 8600737].

11. Myklebust G, Engebretsen L, Braekken IH, Skjolberg A, Olsen OE, Bahr R. Prevention of anterior cruciate ligament injuries in female team handball players: a prospective intervention study over three seasons. Clin J Sport Med. 2003;13(2):71-8. [PubMed: 12629423].

12. Reiman MP, Lorenz DS. Integration of strength and conditioning principles into a rehabilitation program. Int J Sports Phys Ther. 2011;6(3):241-53. [PubMed: 21904701].

13. Lee BK, Nam SW. Rupture of posterior cruciate ligament: diagnosis and treatment principles. Knee Surg Relat Res. 2011;23(3):135-41. doi: 10.5792/ksrr.2011.23.3.135. [PubMed: 22570824].

14. Plos Medicine Editors . Observational studies: getting clear about transparency. PLoS Med. 2014;11(8):ee1001711. doi: 10.1371/journal.pmed.1001711. [PubMed: 25158064]. 